\title{
TOTAL VARIATION WITH GRADIENT HISTOGRAM: A NOVEL APPROACH TO IMAGE DENOISING
}

\author{
Renuka Devi S M \\ ${ }^{I}$ Associate Professor, ECE Department GNITS, Hyderabad- 500050
}

\begin{abstract}
Images play important role in digital world for various research oriented applications and in day to day life, but these images are often available in defective conditions due to noise the gets mixed up with them while capturing and transmitting. Removal of noise from these defective images for better quality plays crucial role, and can be done by using various methods. Natural images have high total variation in them, that is, the integral of the absolute gradient of the signal is high. Image denoising done using Non-linear Total variation (TV) method smoothens the denoised image. To overcome this, a novel gradient histogram method is included in TV method to preserve the fine details of image. Experiments are conducted with various texture structures of image and proved that this method improves the quality of image when compared to earlier method.
\end{abstract}

Keywords: Total Variation, Total Variation Gradient Histogram, Signal Noise Ratio, Structural SIMilarity (SSIM), Feature Similarity (FSIM)

\section{HEADING 1}

Now a days daily life has lots of digital image processing applications they includes digital cameras, intelligent traffic monitoring, handwriting recognition on checks, signature validation, medical research, scientific and space research and so on. However, the images that are used by these applications are available in noisy form. Noise is random changes in intensities of image. Noise is unavoidable and gets into image while capturing or during transmission. There are many types of noises that an image can be effected by; some noises are added to intensities of image such as additive white gaussian noise (AWGN) while others gets multiplied such as poission noise. Added noises are easy to be removed when compared to multiplicative ones. In this paper we consider AWGN with known standard deviation. Earlier noises are removed using filters, but the quality of image is very less with this filters. After filters transform domain methods are used.

Non-linear Total Variation (TV) [1] is first gradient based denoising method used for image noise removal. TV regularization is widely used in image restoration problems for its capability to preserve edges. This TV minimization [1] method was introduced by Rudin, Osher and Fatemi. It is based on the principle that signals with more and possibly counterfeit detail have high total variation, that is, the integral of the absolute gradient of the signal is high. According to this principle, reducing the total variation of the signal subject to it being a close match to the original signal, removes unwanted detail while preserving important details such as edges.

The original image $\mathrm{u}$ is supposed to have a simple geometric description, namely a set of connected sets, the objects, along with their smooth contours, or edges. The image is smooth inside the objects but jumps across the boundaries
At time of its development this method achieved better results when compared to filter and transform domain methods. As the research and development progressed, various methods are being developed for denoising and also the quality of denoised image is also improved a lot. Later studies revealed the fact that, the gradient histogram [5], [6] of images helps a lot in preserving the texture of images while denoising. This motivated us to use gradient histogram with Total variation (TV) method.

\section{TOTAL VARIATION DENOISING WITH} GRADIENT HISTOGRAM:

This method is proposed based on the facts gradients histograms helps very much in preserving the texture of images. Earlier TV method smoothens the denoised image which can be overcome to some extent with this method.

The denoising procedure is done in three stages.

1) K-means segmentation of noisy image

2) Gradient histogram estimation

3) TV denoising.

\subsection{K-Means Segmentation:}

An image contains different textures; similar textures of an image are grouped as region. Thus the image is segmented into different regions, and denoising is applied for individual region instead of for entire image for better results. Image segmentation is one of the mostly used methods to classify the pixels of an image correctly in a decision oriented application. It divides an image into a number of discrete regions such that the pixels have high similarity in each region and high contrast between regions. It is a valuable tool in many field including health care, image processing, traffic image, pattern recognition etc. Image segmentation can be done by using different techniques like threshold 
based, edge based, cluster based, neural network based. Among the various the techniques that are available, one of the most efficient method is the clustering method. One of most used clustering algorithm is k-means clustering, as it is simple and computationally faster.

Different regions in an image can be separated out using Kmeans clustering. This process is known as $\mathrm{K}$-means segmentation. K-means uses an iterative algorithm that minimizes the sum of distances from each object to its cluster centroid, over all clusters. K-means algorithm can be divided into two separate stages. In the first stage it calculates the $\mathrm{k}$ centroid and in the second stage it takes each point to the cluster which has nearest centroid from the respective data point. There are different methods to define the distance of the nearest centroid and one of the most used methods is Euclidean distance. K-means clustering aims to partition $\mathrm{n}$ observations into $\mathrm{k}$ clusters in which each observation belongs to the cluster with the nearest mean, serving as a prototype of the cluster. The function K-means partitions data into $\mathrm{K}$ mutually exclusive clusters, and returns the index of the cluster to which it has assigned each observation. This algorithm moves objects between clusters until the sum cannot be further decreased. Depending on texture features, the image is segmented into regions. The algorithm assumes that the data features form a vector space and tries to find natural clustering in them. The image is segmented into regions based on cluster number. Here in this method of TV with gradient histogram method the image high pass filter coefficients are used data points, which are used to segment the image into different regions.

\subsection{Gradient Histogram Estimation:}

These segmented regions are taken and reference gradient histogram is calculated for each region. In the GHP [2] method the reference gradient histogram is computed from the noisy image itself instead of original image, as original image is not available in real time applications. A regularized deconvolution [3], [7] model is used for computing reference gradient histogram. The normalized histogram of $\nabla x$ can be regarded as a discrete approximation of the probability density function (PDF) of $\mathrm{x}$. For the AWGN v, we can readily model its elements as the samples of an i.i.d. variable. The normalized histogram of $\nabla \mathrm{v}$ can be regarded as a discrete approximation of the probability density function (PDF) of $\epsilon$.

Since $y=x+v$, it is ready to model $\nabla y$ as i.i.d. variable. Let $p_{x}$ be the PDF of $x$, and $p_{y}$ be the PDF of $y$. Since $x$ and $\varepsilon$ are independent, the joint PDF $\mathrm{p}(\mathrm{x}, \varepsilon)$ is

Then the PDF $\mathrm{p}_{\mathrm{y}}$

$$
\mathrm{p}(\mathrm{x}, \varepsilon)=\mathrm{p}_{\mathrm{x}} \times \mathrm{p}_{\varepsilon}
$$

$$
\mathrm{p}_{\mathrm{y}}(\mathrm{y}=\mathrm{t})=\int_{\mathrm{a}} \mathrm{p}_{\mathrm{x}}(\mathrm{x}=\mathrm{a}) \times \mathrm{p}_{\varepsilon}(\varepsilon=(\mathrm{t}-\mathrm{a})) \mathrm{da}
$$

If we use the normalized histogram $h_{x}$ and $h_{y}$ to approximate $\mathrm{p}_{\mathrm{x}}$ and $\mathrm{p}_{\mathrm{y}}$, we can rewrite the above equation in the discrete domain as:

$$
\mathrm{h}_{\mathrm{y}}=\mathrm{h}_{\mathrm{x}} \otimes \mathrm{h}_{\varepsilon}
$$

Where $\otimes$ denotes the convolution operator. Note that $h_{\varepsilon}$ can be obtained by discretizing $\mathrm{p}_{\varepsilon}$, andh $\mathrm{y}$ can be computed directly from the noisy observation $y$.

Obviously, the estimation of $h_{x}$ can be generally modeled as a deconvolution problem:

$$
\mathrm{h}_{\mathrm{r}}=\arg \min _{\mathrm{h}_{\mathrm{x}}}\left\{\left\|\mathrm{h}_{\mathrm{y}}-\mathrm{h}_{\mathrm{x}} \otimes \mathrm{h}_{\varepsilon}\right\|^{2}+\mathrm{c} \cdot \mathrm{R}\left(\mathrm{h}_{\mathrm{x}}\right)\right\}
$$

Where $\mathrm{c}$ is a constant and $\mathrm{R}\left(\mathrm{h}_{\mathrm{x}}\right)$ is some regularization term based on the prior information of natural image's gradient histogram. For region based Gradient histogram method, the regularized deconvolution method can be directly applied to each region to estimate the corresponding reference gradient histogram. The noisy image gradient histogram is computed and it is equalized [4] with reference gradient histogram. The reference gradient histogram is similar to original image.

2.3 Total variation denoising: This equalized noisy image is denoised using total variation method. This method reduces the noise in image by minimizing the gradient changes in image subjected to some constraints. If original image is $u$, and the AWGN noise added to image is $n$, then noisy image can be modeled as $\mathrm{v}=\mathrm{x}+\mathrm{n}$. The constraints imposed while denoising depends on standard deviation value and the constraints are as below

$$
\int_{\Omega}(u(x)-v(x)) d x=0 \text { And } \int_{\Omega}(u(x)-v(x)) d x=\sigma^{2}
$$

The solution $u$ must be as regular as possible in the sense of the total variation, while the difference $\mathrm{v}(\mathrm{x})-\mathrm{u}(\mathrm{x})$ is treated as an error, with a prescribed energy. The constraints prescribe the right mean and variance to $(\mathrm{u}-\mathrm{v})$, but do not ensure that it be similar to a noise

\section{RESULTS}

The quality of image is evaluated by calculating Signal to Noise Ratio (SNR) values and Structural Similarity (SSIM) values and Feature Similarity (FSIM) values.

The structural similarity gives the structural information of image. Its values define how similar two images structurally similar to each other. The formula used to calculate SSIM [8] is as follows

$$
\operatorname{SSIM}(u, v)=\frac{\left(2 \mu_{x} \mu_{y}+C_{1}\right)\left(2 \sigma_{x y}+C_{2}\right)}{\left(\mu_{x}^{2}+\mu_{y}^{2}+C_{1}\right)+\left(\sigma_{x}^{2}+\sigma_{y}^{2}+C_{2}\right)}
$$

The FSIM [9] defines how much of features of two images are similar to each other. The formula used to computed FSIM is as follows

$$
\operatorname{FSIM}(u(x), v(x))=\frac{\sum_{x \in \Omega} S_{L}(x) \cdot P_{m}(x)}{\sum_{x \in \Omega} P C_{m}(x)}
$$




\subsection{Comparative Results of TV and TVGH}

\section{Methods}

The results of two methods are conducted on four natural images with different texture structures and with different standard deviation values of 10 to 30 .

\begin{tabular}{|l|l|l|l|l|l|l|}
\hline \multirow{3}{*}{ Image name } & \multicolumn{5}{|c|}{ SNR values (dB) } \\
\cline { 2 - 7 } & \multicolumn{2}{|c|}{$\sigma=10$} & \multicolumn{2}{c|}{$\sigma=20$} & \multicolumn{2}{c|}{$\sigma=30$} \\
\cline { 2 - 7 } & TV & TVGH & TV & TVGH & TV & TVGH \\
\hline Cameraman & 24.284 & 24.30 & 22.6946 & 22.709 & 20.037 & 20.091 \\
\hline Puppy & 26.52 & 26.558 & 24.8995 & 24.914 & 21.984 & 22.013 \\
\hline Cars & 21.969 & 21.980 & 21.1666 & 21.18 & 19.441 & 19.451 \\
\hline Monarch_full & 24.861 & 24.906 & 22.4812 & 22.487 & 19.49 & 19.47 \\
\hline
\end{tabular}

\begin{tabular}{|l|l|l|l|l|l|l|}
\hline \multirow{3}{*}{ Image name } & \multicolumn{6}{|c|}{ SSIM values } \\
\cline { 2 - 7 } & \multicolumn{2}{|c|}{$\sigma=10$} & \multicolumn{2}{c|}{$\sigma=20$} & \multicolumn{2}{c|}{$\sigma=30$} \\
\cline { 2 - 7 } & TV & TVGH & TV & TVGH & TV & TVGH \\
\hline Cameraman & 0.8658 & 0.8662 & 0.7817 & 0.7834 & 0.6007 & 0.6042 \\
\hline Puppy & 0.8138 & 0.8143 & 0.7531 & 0.7531 & 0.6040 & 0.6050 \\
\hline Cars & 0.8287 & 0.8289 & 0.7639 & 0.7644 & 0.6217 & 0.6237 \\
\hline Monarch_full & 0.9232 & 0.9257 & 0.8541 & 0.8528 & 0.7211 & 0.7108 \\
\hline
\end{tabular}

\begin{tabular}{|l|l|l|l|l|l|l|}
\hline \multirow{3}{*}{ Image name } & \multicolumn{4}{|l|}{ FSIM values } \\
\cline { 2 - 7 } & \multicolumn{2}{|c|}{$\sigma=10$} & \multicolumn{2}{c|}{$\sigma=20$} & \multicolumn{2}{c|}{$\sigma=30$} \\
\cline { 2 - 7 } & TV & TVGH & TV & TVGH & TV & TVGH \\
\hline Cameraman & 0.9006 & 0.9008 & 0.8771 & 0.8779 & 0.8082 & 0.8113 \\
\hline Puppy & 0.9577 & 0.9578 & 0.9389 & 0.9389 & 0.8951 & 0.8962 \\
\hline Cars & 0.9782 & 0.9783 & 0.9570 & 0.9565 & 0.9193 & 0.9206 \\
\hline Monarch_full & 0.9367 & 0.9367 & 0.9042 & 0.9019 & 0.8346 & 0.8302 \\
\hline
\end{tabular}
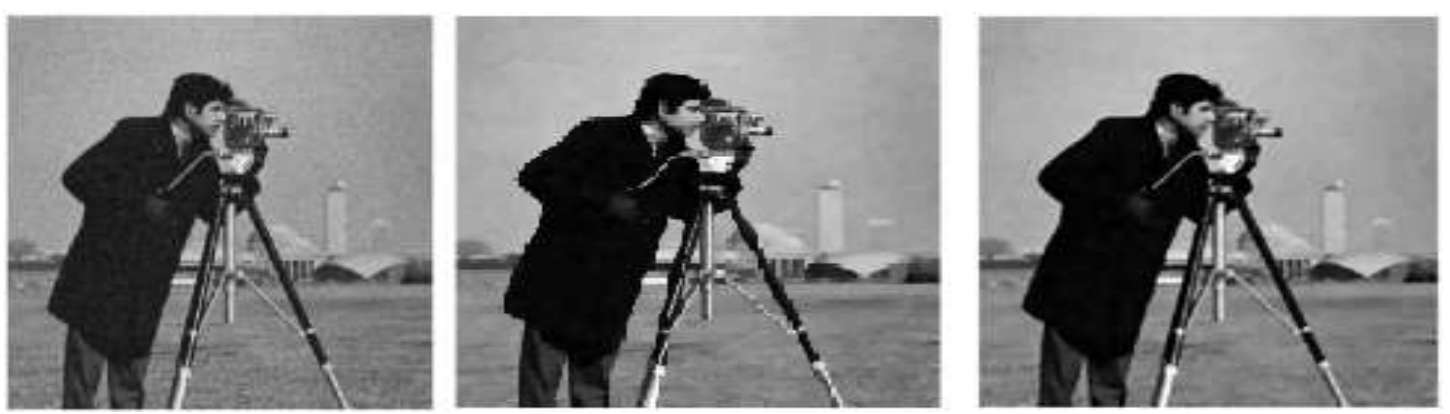

Fig 3.1 Noisy image with standard deviation of 10 and denoised images of TV and TVGH methods 

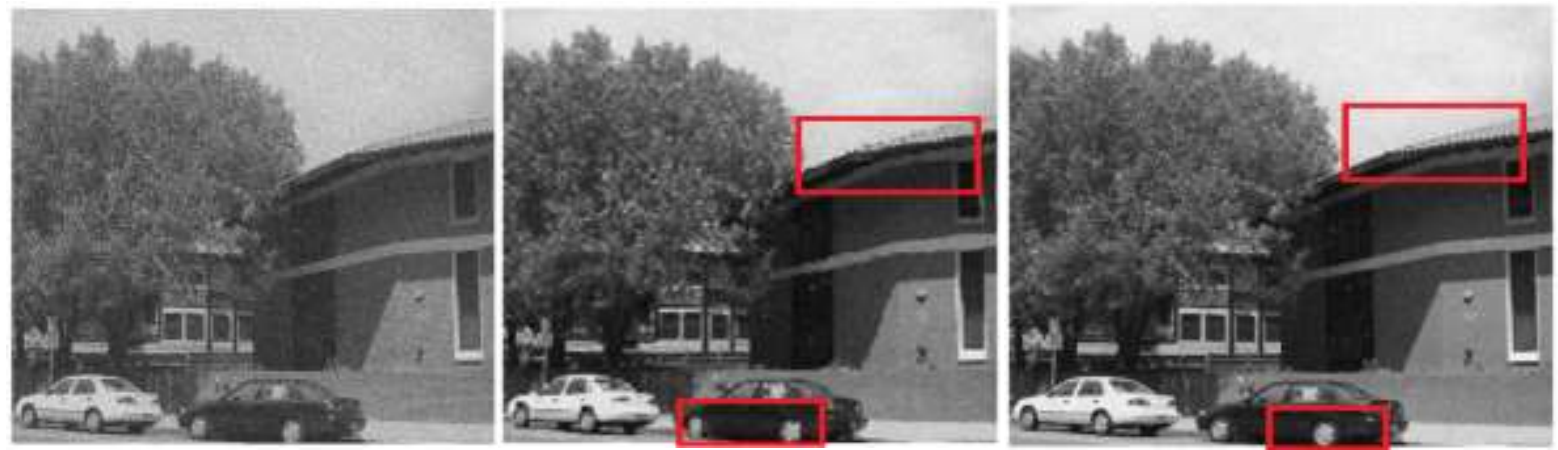

Fig 3.2 Noisy and Denoised images $(\sigma=20)$ of TV and TVGH methods TVGH methods
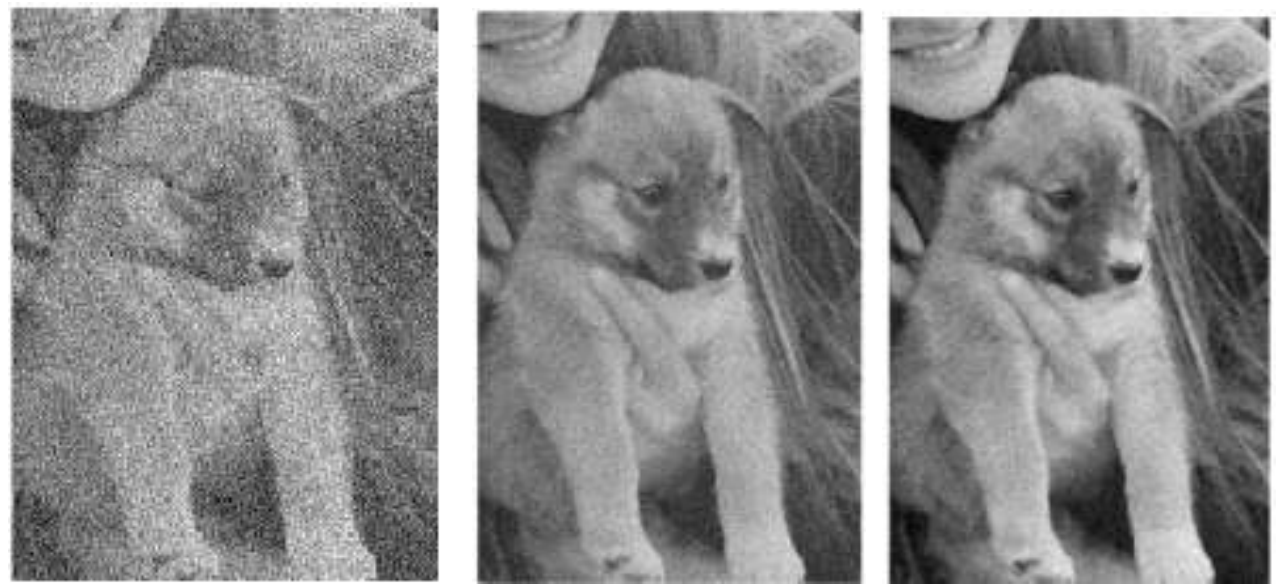

Fig 3.2 Noisy and Denoised images $(\sigma=40)$ of TV and TVGH methods

We can clearly see the difference in denoised images done using TV and TVGH methods. The image part in red boxes clearly shows that texture can be preserved by using gradient histogram.

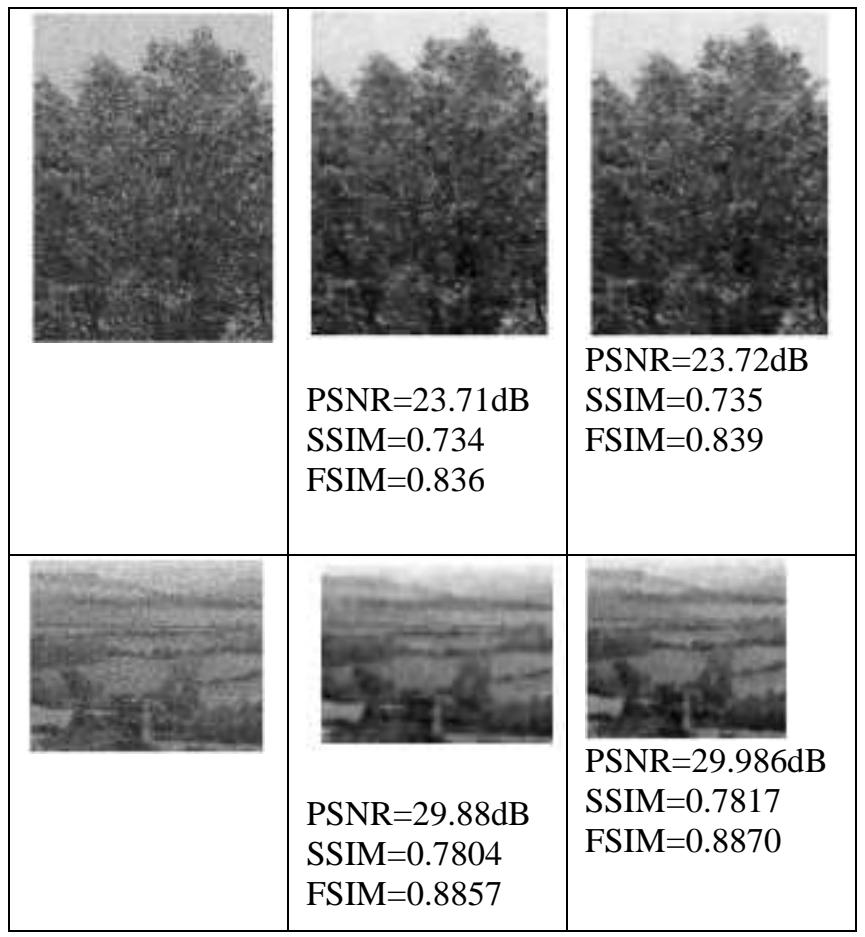

Fig 3.3 cropped patches of noisy and denoised images using TV and TVGH methods

\section{CONCLUSION}

Gradient histogram is new method applied to total variation method. Gradient histogram can improve a little extent of the SNR values, but preserves the texture of denoised images to some extent. The results of TVGH are not up to mark, when compared with latest methods but are better with that of TV. The feature similarity values and structure similarity values are improved, but can be further improved.

\section{REFERENCES}

[1] L. Rudin, S. Osher, and E. Fatemi, "Nonlinear total variation based noise removal algorithms," Phys. D, Nonlinear Phenomena, vol. 60, nos. 1-4,pp. 259-268, Nov. 1992.

[2] W. Zuo, L. Zhang, C. Song, and D. Zhang, "Texture Enhanced Image Denoising via Gradient Histogram Preservation," in Proc. Int. Conf. Compu. Vis. Pattern Recognit., 2013.

[3] D. Krishnan, R. Fergus, "Fast image deconvolution using hyper- Laplacian priors," in Proc. Neural Inf. Process. Syst., pp. 1033-1041,2009.

[4] S. M. Pizer, E. P. Amburn, J. D. Austin, R. Cromartie, A. Geselowitz, T. Greer, B. H. Romeny, J. B. Zimmerman, and K. Zuiderveld, "Adaptive histogram equalization and its variations," Comp. Vis. Graph. Image Process., vol. 39, no. 3, pp. 355-368, Sep. 1987.

[5] B. Julesz, "Textons, the elements of texture perception, and their interactions," Nature, vol. 290, pp. 91-97, Mar. 1981. 
[6] J. A. Snyman, Practical Mathematical Optimization: An Introduction to Basic Optimization Theory and Classical and New Gradient-Based Algorithms, Springer, 2005.

[7] V. Jain and H. Seung, "Natural image denoising with convolutional networks," in Proc. Neural Inf. Process.Syst., pp. 769-776, 2008.

[8] Z. Wang, A. C. Bovik, H. R. Sheikh, and E. P. Simoncelli, "Image quality assessment: from error visibility to structural similarity," IEEE Trans. Image Process., vol. 13, no. 4, pp. 600-612, Apr. 2004.

[9] L. Zhang, L. Zhang, X. Mou, and D. Zhang, "FSIM: a feature similarity index for image quality assessment," IEEE Trans. Image Process., vol. 20, no. 8, pp. 2378C2386, Aug. 2011. 